Rabaska

Revue d'ethnologie de l'Amérique française

Piette, Albert.Petit traité d'anthropologie, [Charleroi], Socrate Éditions Promarex, "Science éphémère " $n^{0} 3$, [2006], 95 p. ISBN 2-930394-07-2

\title{
Vanessa Ferey
}

Volume 8, 2010

URI : https://id.erudit.org/iderudit/045289ar

DOI : https://doi.org/10.7202/045289ar

Aller au sommaire du numéro

Éditeur(s)

Société québécoise d'ethnologie

ISSN

1703-7433 (imprimé)

1916-7350 (numérique)

Découvrir la revue

Citer ce compte rendu

Ferey, V. (2010). Compte rendu de [PIETTE, ALbERT.Petit traité d'anthropologie, [Charleroi], Socrate Éditions Promarex, « Science éphémère " $n^{0}$ 3, [2006], 95 p. ISBN 2-930394-07-2]. Rabaska, 8, 237-239. https://doi.org/10.7202/045289ar d'utilisation que vous pouvez consulter en ligne.

https://apropos.erudit.org/fr/usagers/politique-dutilisation/ 
Piette, Albert. Petit traité d'anthropologie, [Charleroi], Socrate Éditions Promarex, « Science éphémère » n 3, [2006], 95 p. ISBN 2-930394-07-2

Fort d'un argumentaire en quatre points, Albert Piette nous dépeint dans ce court essai une critique de l'anthropologie tout aussi passionnée qu'originale. En effet, l'anthropologue et professeur de l'Université d'Amiens nous fait part d'une réflexion captivante à propos des spécificités de la discipline et de la double interrogation qu'elle suscite, concernant la question mystérieuse des origines, mais aussi celle de la situation réelle. Ces « leçons », tel qu'il le mentionne, s'adressent d'abord aux étudiants en sciences sociales. Elles visent à montrer que « la destinée de l'anthropologie n'est pas nécessairement la socio-ethnologie », tout en annonçant la possibilité de revenir aux questions fondamentales de la spécificité humaine.

Dès lors, il rappelle que le projet de l'anthropologie est « une connaissance globale de l'homme » et en particulier «des propriétés générales de la vie sociale » pour ne citer que Lévi-Strauss. Il fait aussi remarquer en introduction que cette discipline s'appuie sur l'étude des phénomènes sociaux et ainsi affiche une position particulière " au carrefour de différentes disciplines ». Cependant les anthropologues auraient abandonné la question de l'origine aux philosophes, laissant ainsi leur science devenir synonyme de celle de la sociologie ou de l'ethnologie en se focalisant sur «les spécificités d'un événement, d'une activité, d'un groupe » et laissant de côté l'étude fondamentale des invariants entre les hommes (p. 10). Or, l'anthropologie ne doit pas être « cantonnée à l'étude des diversités socio-culturelles » en demeurant indifférente à la question des origines qui s'avère être la limite de cette science, mais aussi le fondement de son projet : «comprendre l'être humain ». La dichotomie, origines vs réel, sera d'ailleurs exacerbée sous une pléiade de courants classiques en anthropologie afin de mettre en perspective cette dualité et ainsi mesurer les limites et les insuffisances des théorisations de ses pairs. L'auteur débutera par un rappel des « scénarios d'origine » afin de guider le lecteur dans la réflexion à venir sur les exposés édificateurs de la discipline. Ainsi le récit mythique de Protagoras va lui servir de point d'appui afin de citer les trois caractéristiques essentielles à l'hominisation : le langage, l'outil et la règle. Triade que l'on retrouvera tout au long de l'ouvrage et à travers laquelle différents travaux seront mobilisés.

De ce fait, quatre conceptions arrivent à l'avant-plan de son argumentaire afin d'apporter chacune une réponse spécifique. Il sera mis en relief sous trois mots-clés pour chaque référence : l'évolutionnisme de Morgan : « idées, subsistances et inventions », le fonctionnalisme de Malinowski : «besoins, culture et famille » et le structuralisme de Lévi-Strauss : « cerveau, prohibition et échange ». En dernier lieu, les travaux sur le projet de l'anthropologie 
fondamentale de Godelier : « continuité, alliance et descendance » tenteront eux aussi de répondre au problème suivant : «Comment l'anthropologie vat-elle penser l'humanité primitive ?» (p. 15).

L'exemple de Morgan dégage ainsi l'idée que l'accès permanent à la nourriture semble être une des clés de la réussite de l'évolution des hommes. Par la suite, Malinoswki met en avant le besoin et la nécessité d'un système organisé d'interdits sexuels pour créer un mécanisme de protection du lien social. De son côté, Lévi-Strauss valorise la grande transformation opérée grâce au développement des fonctions cérébrales de l'homme qui réside dans l'instauration des règles en particulier négatives et de leurs conséquences sur l'établissement de liens sociaux. Quant à Godelier, il tente de cerner l'essence de l'homme et ce qu'il y a d'humain en lui et présente l'interdit de l'inceste comme une transformation de mécanismes déjà existants dans la vie de certains animaux.

À la lumière de ces traditions disciplinaires s'éclaircira le reproche principal et non des moindres de l'essai : l'oubli phénoménologique de l'étude ethno-anthropologique qui devrait assumer l'idée que " l'homme des commencements est bel et bien un homme socio-culturel» (p. 30). Ce cadre factuel de « l'homme socio-culturel» floue les recherches de l'anthropologue menant des recherches sur les origines de l'être humain et véhiculant un « déficit anthropologique» nuisant à l'étude. «N'est-on pas allé trop loin dans cette direction socio-culturaliste ? \elon Piette, l'anthropologie réalise sous une telle réflexion une "dimension épistémologique critique » par rapport aux modèles de pensée des sciences sociales.

Cette critique de la " sociologie programmée » passera d'une part par les travaux de Durkheim et Weber afin de décortiquer deux principes : «l'objet de l'analyse et sa nature collective ». D'autre part, l'anthropologie des actions associant l'homme socio-culturel à un ensemble de compétences et d'états sera étudiée par une série de logiques d'action en passant par les travaux de Bourdieu, Goffman, Thévenot, Pharo, Garfinkel, Husserl, Bateson et bien d'autres. Cette partie fera alors apparaître trois risques de la théorie sociologique : "écraser les actions sous un modèle, un paradigme, une métaphore ; travailler trop exclusivement à partir de situation limites $[\ldots]$; focaliser son attention et son analyse sur un seul monde, plutôt que de suivre les acteurs dans l'hétérogénéité des mondes [...]». L'homme socio-culturel dépeint dans cette partie ne correspond pas à toutes les compétences exercées par l'être humain en situation, c'est pourquoi il s'avère « anthropologiquement impossible » (p. 41). L'auteur conclut par un questionnement qui annonce la suite de l'essai : "Comment faire des sciences sociales sans pratiquer la sociologie ou l'ethnologie ? » Piette annonce alors qu'une dynamique féconde entre ethnographie et anthropologie constitue une solution possible. 
Quelques propositions vont suivre la question : «Comment à la fois maintenir le cadre de la discipline sociologique ou ethnologique [...] et décrire le comportement des êtres humains observés dans leur singularité ? » Afin de sortir du vase clos de l'étude épistémologique, Piette annonce que c'est précisément «le mode d'être des hommes que l'ethnographie pourrait redécouvrir » et suggère la pratique d'un " décentrement par rapport aux modalités habituelles de la mise en perspective socio-culturaliste $»$ (p. 49). L'exemple des travaux de Paul Veyne cherchant à décaper la réalité de l'emphase de son discours descriptif et interprétatif habituel soulève un paradoxe : celui du « mode mineur de la réalité ». C'est visiblement à travers lui qu'il importerait de repérer empiriquement, de construire théoriquement et de décrire le fait social en tant qu'il est « minimalement socialisé » (p. 54). Finalement, la partie concernant l'anthropologie entre les êtres prouve que « c'est moins l'outil, le langage, la société qui font la caractéristique humaine que l'avènement d'une forme d'extériorité à la situation». Elle semble être le point de convergence, le fil qui « se tisse entre chacun et se constitue en indice d'humanité ». Ainsi, les « petites choses [...] sont ce qui révèlent au mieux la présence humaine de l'homme au monde : une présence dégagée par rapport à la situation, à l'interaction et l'enjeu de sens qui s'y joue » (p. 80). Il conclura sur le fait qu'il est alors impératif que « la sociologie et l'ethnologie se posent [...] en une actualogie socio-culturelle », tout comme il est nécessaire que l'anthropologie «ne suspende pas sa réflexion sur la nature de l'homme ».

Albert Piette dévoilera entièrement sa passion dans un dernier chapitre, en évoquant les liens tissés entre le savoir anthropologique et sa propre vie. Loin d'être un essai au discours prétentieux d'avertissement, le récit du professeur est avant tout une démonstration pédagogique brillante et il encourage par cette belle leçon sur l'humanité la réflexion autour du dilemme anthropologique.

VANesSa Ferey

Université du Québec à Montréal

Postic, Fañch (sous la direction de). Bretagnes. Du cour aux lèvres. Mélanges offerts à Donatien Laurent. Rennes, Presses universitaires de Rennes, «Essais », 2009, 421 p. ISBN 978-2-7535-0767-8.

Je n'ai jamais visité la Bretagne. Je n'en ai qu'une collection d'images disparates accumulées au hasard de mes lectures qu'une curiosité vagabonde s'est plu à assembler en un tableau qui tient davantage du kaléidoscope que du travail du peintre. Dans ce bric-à-brac figurent, pêle-mêle, Anne de 